DFPD 00/TH/50

UNICAL-TH 00/8

November 2000

\title{
ANALYTIC MODEL OF REGGE TRAJECTORIES •
}

\author{
R. Fiore ${ }^{a \dagger}$, L.L. Jenkovszky ${ }^{b \S}$, V. Magas ${ }^{b, c \ddagger}$, F. Paccanoni ${ }^{d *}$ and A. Papa ${ }^{a \dagger}$ \\ ${ }^{a}$ Dipartimento di Fisica, Università della Calabria, \\ Istituto Nazionale di Fisica Nucleare, Gruppo collegato di Cosenza \\ I-87036 Arcavacata di Rende, Cosenza, Italy \\ ${ }^{b}$ Bogolyubov Institute for Theoretical Physics, \\ National Academy of Sciences of the Ukraine \\ 03143 Kiev, Ukraine \\ ${ }^{c}$ Department of Physics, Bergen University \\ Allegaten 55, N-5007, Norway \\ ${ }^{d}$ Dipartimento di Fisica, Università di Padova, \\ Istituto Nazionale di Fisica Nucleare, Sezione di Padova \\ via F. Marzolo 8, I-35131 Padova, Italy
}

\begin{abstract}
A model for a Regge trajectory compatible with the threshold behavior required by unitarity and asymptotics in agreement with analyticity constraints is given in explicit form. The model is confronted in the time-like region with widths and masses of the mesonic resonances and, in the spacelike region, the $\rho$ trajectory is compared with predictions derived from $\pi-N$ charge-exchange reaction. Breaking of the exchange degeneracy is studied in the model and its effect on both the masses and widths is determined. PACS numbers: 11.55.Jy, 11.55.Bq, 14.40.-n.
\end{abstract}

${ }^{\diamond}$ Work supported by the Ministero italiano dell'Università e della Ricerca Scientifica e Tecnologica and by the INTAS.

$\dagger$ e-mail address: FIORE,PAPA @CS.INFN.IT

$\S$ e-mail address: JENK @GLUK.ORG

${ }^{\ddagger} e$-mail address: VLADIMIR @FI.UIB.NO

*e-mail address: PACCANONI @PD.INFN.IT 


\section{Introduction}

Recently a lot of activity has been dedicated to various studies of the Regge trajec-

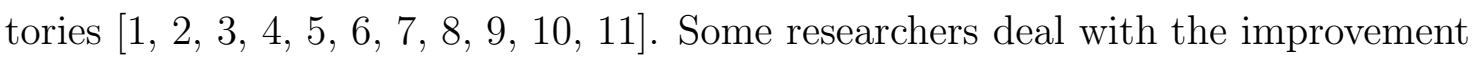
and extension of the existing fits to hadronic total cross sections by including, apart from the slopes and intercepts of degenerate or non-degenerate linear Regge trajectories, also the relevant Chew-Frautschi plots [7, 8, 9]. The most complete and updated Chew-Frautschi plots for various (linear) trajectories can be found in paper [5]. The essential role of the non-linearity was recognized and scrutinized in the papers [2, 3, 国, where studies of the analytic and asymptotic properties of the trajectories were also performed. The connection of the Regge trajectories with dynamical models, based on non-relativistic potentials, hadronic strings, dual models etc. was studied recently in Refs. [四, [1].

The basic problem in constructing Regge trajectories is to combine the nearly linear rise of the real part in the whole range of observed resonances with the presence of a sizable imaginary part. The combination of these properties in a single analytic function seems to be difficult, if not impossible, unless the linear part stops rising at larger values of the argument. This is true for the model we present below.

Besides the experimental evidence, the dominant idea about the continuous rising linear trajectories found strong support from the narrow-resonance (e.g. Veneziano) dual models, later replaced by hadronic strings. The "narrow resonance width" was treated in those models as an approximation, where resonances' widths are considered negligible in comparison with the spacing between the neigh-

bors. The data do not support this idea, thus ruling out the concept of continuously rising linear trajectories. 
Exchange degeneracy (EXD) of the Regge trajectories (and the residues) with opposite signatures is an approximate symmetry based both on observations and theoretical speculations (the quark model, $S U(3)$ symmetry, duality). It is attractive as it relates various objects (trajectories, spectra of particles, residues, cross sections) reducing thus the number of the free parameters. The extent to which this symmetry is violated has been (and is still) debated ever since this symmetry was discovered. It depends on the considered reaction (trajectory) and the required precision (at the cost of the number of the free parameters introduced). In our analysis we consider both cases - exact EXD as well as its violation.

There are two basic theoretical restrictions, which must be satisfied by Regge trajectories - the threshold and asymptotic behaviour. The behaviour at the threshold is determined by unitarity and was studied in Refs. 112, 13, 14, 15. On the other hand, only bounds exist on the asymptotic behaviour of the trajectories. Analyticity sets an upper bound [13] and there are some plausible arguments [16, 17, 18, 19] in favour of a stronger upper bound. They suggest a square root asymptotic behaviour on the physical sheet, $\alpha(s) \sim(-s)^{1 / 2}$, for $|s| \rightarrow \infty$, implying, as we shall see, that the real part of the trajectories is bounded by a constant, i.e. that resonances "terminate" at some point.

In the present paper we construct an explicit model for light unflavored mesonic trajectories satisfying the above properties, i.e. our model has correct threshold and asymptotic behaviour and fits the data on both the masses and widths of the observed mesonic resonances. We did not include baryonic resonances, whose widths seem to show a regularity, $\operatorname{Im} \alpha(s) \sim \operatorname{Re} \alpha(s)$, not shared by mesonic resonances [2, [16]. 
We apply our analysis of the meson trajectories to both the positive (particles spectra) and negative (scattering region) values of their arguments (we denote it by $s$, although our results are crossing symmetric in the Mandelstam variables $s, t, u$ ) by fitting the parameters of the $\rho-a_{2}$ exchange degenerate trajectory to $\pi-N$ charge-exchange reaction data as in [20].

Although various aspects of mesonic trajectories have been already studied and clarified in several papers [2, 16, 17, 18, 19] we are not aware of any complete and coherent solution of the problem, satisfying the correct threshold behaviour and fitting the widths of the mesonic trajectories.

\section{The model}

The properties of the trajectories following from analyticity and unitarity have been discussed in Refs. 112, 13, 14, 15, 16]. In the complex $s$-plane the trajectory $\alpha(s)$ has only right-hand branch lines apart from branch points at the intersection with another singularity in the $l$-plane. The requirement that there are no crossing poles and that $\alpha(s)$ is bounded for $s \rightarrow \infty$, leads to a dispersion relation of the form

$$
\alpha(s)=\alpha(0)+\frac{s}{\pi} \int_{0}^{\infty} d s^{\prime} \frac{\mathcal{I} m \alpha\left(s^{\prime}\right)}{s^{\prime}\left(s^{\prime}-s\right)}
$$

where $\operatorname{Im} \alpha(s)>0$ since the pole must lie in the upper half $l$-plane. In other words, no complex singularity of the partial wave exists for real $l$. The boundedness of $\alpha(s)$ for $s \rightarrow \infty$ follows from the assumption that the amplitude $A(s, t)$ is bounded by a polynomial in $t$ and $s$. This restriction is equivalent to the condition that the amplitude, in the Regge form, should have no essential singularity at infinity in the 
cut plane. The condition that $\mathcal{R} e \alpha(s)$ is bounded by a constant, for $s \rightarrow \infty$, leads to

$$
|\alpha(s)|<M s^{q}, \quad \text { for } s \rightarrow \infty
$$

with $q<1$ and $M$ an arbitrary constant. Equation (11) implies that $\mathcal{R} e \alpha(s)$ cannot be exactly linear in $s$ but it does not give any information on the deviation from linearity unless a specific model has been selected for the imaginary part $\mathcal{I} m \alpha(s)$.

Unitarity requires the threshold behaviour

$$
\mathcal{I} m \alpha(s) \propto\left(s-s_{0}\right)^{\mathcal{R} e \alpha\left(s_{0}\right)+1 / 2}
$$

as shown in Ref. [12, 13, 15]. For example, the first right-hand branch point will be at $s_{0}=\left(2 m_{\pi}\right)^{2}$ for the $\rho$ trajectory, and $s_{0}=\left(3 m_{\pi}\right)^{2}$ for the $\omega$. To build an explicit model we must constrain the asymptotic behaviour of the trajectory and find a rule to determine the contribution of several thresholds to $\mathcal{I} m \alpha(s)$. We borrow both properties of the model from Ref. [19]. First, we assume the square-root asymptotic on the physical sheet

$$
|\alpha(s)| \sim s^{\frac{1}{2}}, \quad \text { for } s \rightarrow \infty
$$

which satisfies the condition (2) and is realized in dual models [17, 18]. Secondly, we assume additivity of threshold contributions

$$
\alpha(s)=\alpha(0)+\sum_{n} \alpha_{n}(s)
$$

where $\alpha_{n}(s)$ has only one threshold branch point on the physical sheet. We require this hypothesis, which seems to be plausible, in order to set up a completely soluble model; at least we are not aware of a reasonable alternative. Contrary to [19], we consider in the following only thresholds determined from pions and other mesonic resonances. 
We start from a simple analytical model, where the imaginary part of the trajectory is chosen as a sum of the single threshold terms

$$
\mathcal{I} m \alpha(s)=\sum_{n} c_{n}\left(s-s_{n}\right)^{1 / 2}\left(\frac{s-s_{n}}{s}\right)^{\mathcal{R} e \alpha\left(s_{n}\right)} \theta\left(s-s_{n}\right)
$$

with the correct asymptotic and threshold behaviour. In Eq. (6) all $c_{n}$ 's are positive [13, 15. It should be stressed that this choice is a crude approximation to the complexity of the true imaginary part. For example, the exponent $\mathcal{R} e \alpha\left(s_{n}\right)$ should be a continuous function of $s$.

From the dispersion relation for the trajectory,

$$
\mathcal{R} e \alpha(s)=\alpha(0)+\frac{s}{\pi} P V \int_{0}^{\infty} d s^{\prime} \frac{\mathcal{I} m \alpha\left(s^{\prime}\right)}{s^{\prime}\left(s^{\prime}-s\right)},
$$

where $P V$ means the Cauchy Principal Value of the integral, the real part can be easily calculated [16, 21]. Defining $\lambda_{n}=\mathcal{R} e \alpha\left(s_{n}\right)$ we get:

$$
\begin{aligned}
\mathcal{R} e \alpha(s) & =\alpha(0)+\frac{s}{\sqrt{\pi}} \sum_{n} c_{n} \frac{\Gamma\left(\lambda_{n}+3 / 2\right)}{\Gamma\left(\lambda_{n}+2\right) \sqrt{s_{n}}}{ }_{2} F_{1}\left(1,1 / 2 ; \lambda_{n}+2 ; \frac{s}{s_{n}}\right) \theta\left(s_{n}-s\right)+ \\
& +\frac{2}{\sqrt{\pi}} \sum_{n} c_{n} \frac{\Gamma\left(\lambda_{n}+3 / 2\right)}{\Gamma\left(\lambda_{n}+1\right)} \sqrt{s_{n}}{ }_{2} F_{1}\left(-\lambda_{n}, 1 ; 3 / 2 ; \frac{s_{n}}{s}\right) \theta\left(s-s_{n}\right) .
\end{aligned}
$$

From Eq. (7) we can get the slope

$$
\begin{gathered}
\mathcal{R} e \alpha^{\prime}(s)=\frac{1}{\sqrt{\pi}} \sum_{n} c_{n} \frac{\Gamma\left(\lambda_{n}+3 / 2\right)}{\Gamma\left(\lambda_{n}+2\right) \sqrt{s_{n}}}{ }_{2} F_{1}\left(2,1 / 2 ; \lambda_{n}+2 ; \frac{s}{s_{n}}\right) \theta\left(s_{n}-s\right)+ \\
+\frac{4}{3 \sqrt{\pi}} \sum_{n} c_{n} \frac{\Gamma\left(\lambda_{n}+3 / 2\right)}{\Gamma\left(\lambda_{n}\right)} \frac{s_{n}^{3 / 2}}{s^{2}}{ }_{2} F_{1}\left(1-\lambda_{n}, 2 ; 5 / 2 ; \frac{s_{n}}{s}\right) \theta\left(s-s_{n}\right),
\end{gathered}
$$

since the derivatives of the $\theta$-functions in Eq. (17) cancel. Equations (6) and (8) determine the width of the resonances through the relation

$$
\Gamma\left(M^{2}\right)=\frac{\mathcal{I} m \alpha\left(M^{2}\right)}{M \mathcal{R} e \alpha^{\prime}\left(M^{2}\right)}
$$


where $\Gamma$ is the width of the resonance and $M$ is its mass.

Suppose now that there is a threshold, higher than all resonances, at the position $s_{x}$ and with coefficient $c_{x}$. This threshold will contribute to the real part and to the slope, but not to the imaginary part in the resonance region. Its relevance to the real part of the trajectories and to the widths of the resonance will become clear in the following. Fits of the unknown parameters will be presented and discussed in the next Section. We only notice that Eq. (7) gives the complete real part of the trajectory, both in the time-like and in the space-like regions.

Of particular interest is the behaviour of the trajectory above $s_{x}$. From Eqs. (77 9) we can see that for $s \gg s_{x}$ the $\mathcal{R} e \alpha$ reaches a saturation point and stays nearly constant.

\section{Fitting the resonances masses and widths}

The feature of the real part of trajectory, namely $\mathcal{R} e \alpha(s)$ tends to constant, when $s \rightarrow \infty$, means that the number of resonances lying on it is limited. As noticed before, approximate linearity in the region where experimental data require it, can be attained by introducing a threshold higher than the masses of all known relevant resonances. The highest threshold entails two unknown parameters, its position $s_{x}$ and the coefficient $c_{x}$, and contributes to Eq. (6) only above the resonance region. It can be considered as an effective threshold. This fact suggests an iterative method for the fitting procedure.

A rough estimate of $\mathcal{R} e \alpha\left(s_{n}\right) \equiv \lambda_{n}$, can be obtained from a linear trajectory, adapted to the experimental data. In this way we fix also the lowest order value of 

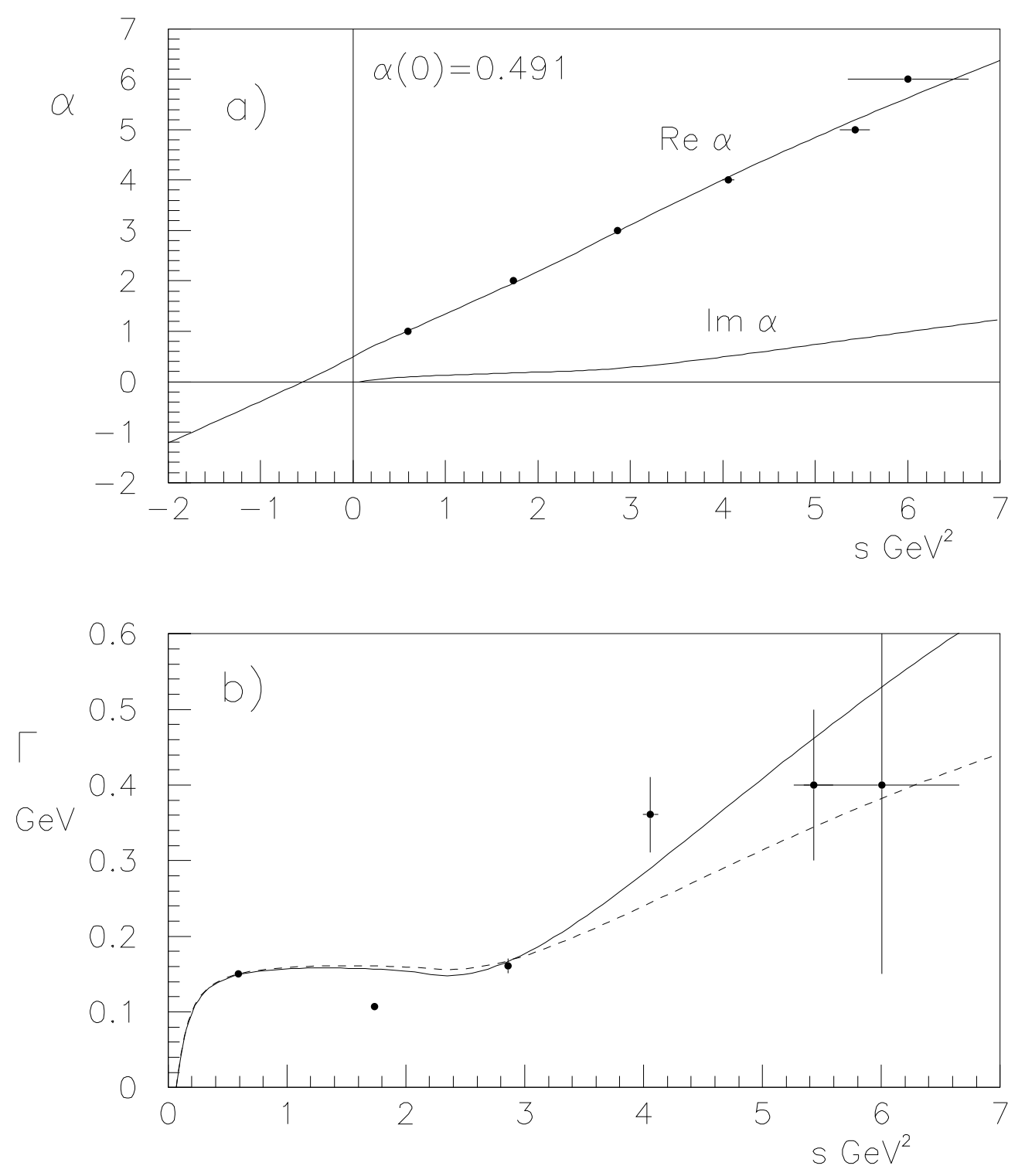

Figure 1: (a) $\mathcal{R} e \alpha(s), \operatorname{Im} \alpha(s)$ for exchange degenerate $\rho-a_{2}$ trajectory; (b) continuous line: $\Gamma(s)$ for the $\rho-a_{2}$ trajectory, dashed line: $\Gamma(s)$ for the nondegenerate $\rho$ trajectory. 
the slope. Intercepts and slopes of mesonic trajectories can be taken, for example, from the accurate analysis in [9]. In addition to the lowest $\pi-\pi$ threshold we will consider in the following the open channels, where one, or both pions, are substituted by mesonic resonances. Once the thresholds $s_{n}$ have been chosen, in accordance with the considered trajectory, Eq. (9) can be used to fit the unknown parameters $c_{n}$ from the experimental masses and widths of the resonances. Then, the fit of the spins of the resonances fix the remaining parameters, $\alpha(0), c_{x}$ and $s_{x}$, from Eq. (7). In the minimization procedure an error can be associated with the spin of a resonance in terms of the error in the mass and the estimated errors for intercept and slope [9]. In such a way we get the first order correction to the trajectory. Iterations can be performed by repeating the previous steps till a stable output is obtained.

The scarcity of the available experimental data, forces an a priori choice of the thresholds. In all cases with positive G-parity, the $\pi-\pi$ threshold will be present, hence $s_{1} \simeq 0.077 \mathrm{GeV}^{2}$ for $\rho$ and $f$ trajectories. After a large number of trials we have chosen $s_{2}$ near $2 \mathrm{GeV}^{2}$ for all trajectories. To be precise, for the $G=+$ trajectories, we set $s_{2}=2.12 \mathrm{GeV}^{2}$ that corresponds to a $\pi-a_{2}(1320)$ threshold. The position of the last threshold, $s_{3} \equiv s_{x}$, does not influence sensibly the result. Hence, if the $\chi^{2}$ prefers a very high $s_{x}$ (we will see later that this is the case for $\rho$ and $\rho+a_{2}$ trajectories), we set $s_{x}=30 \mathrm{GeV}^{2}$. We have tried to insert extra thresholds in a large variety of ways without finding any improvement. By fixing the thresholds we limit the capability of the model in reproducing the experimental data, but, as we will see in the following, this still allows us to obtain a good agreement with experiment. 

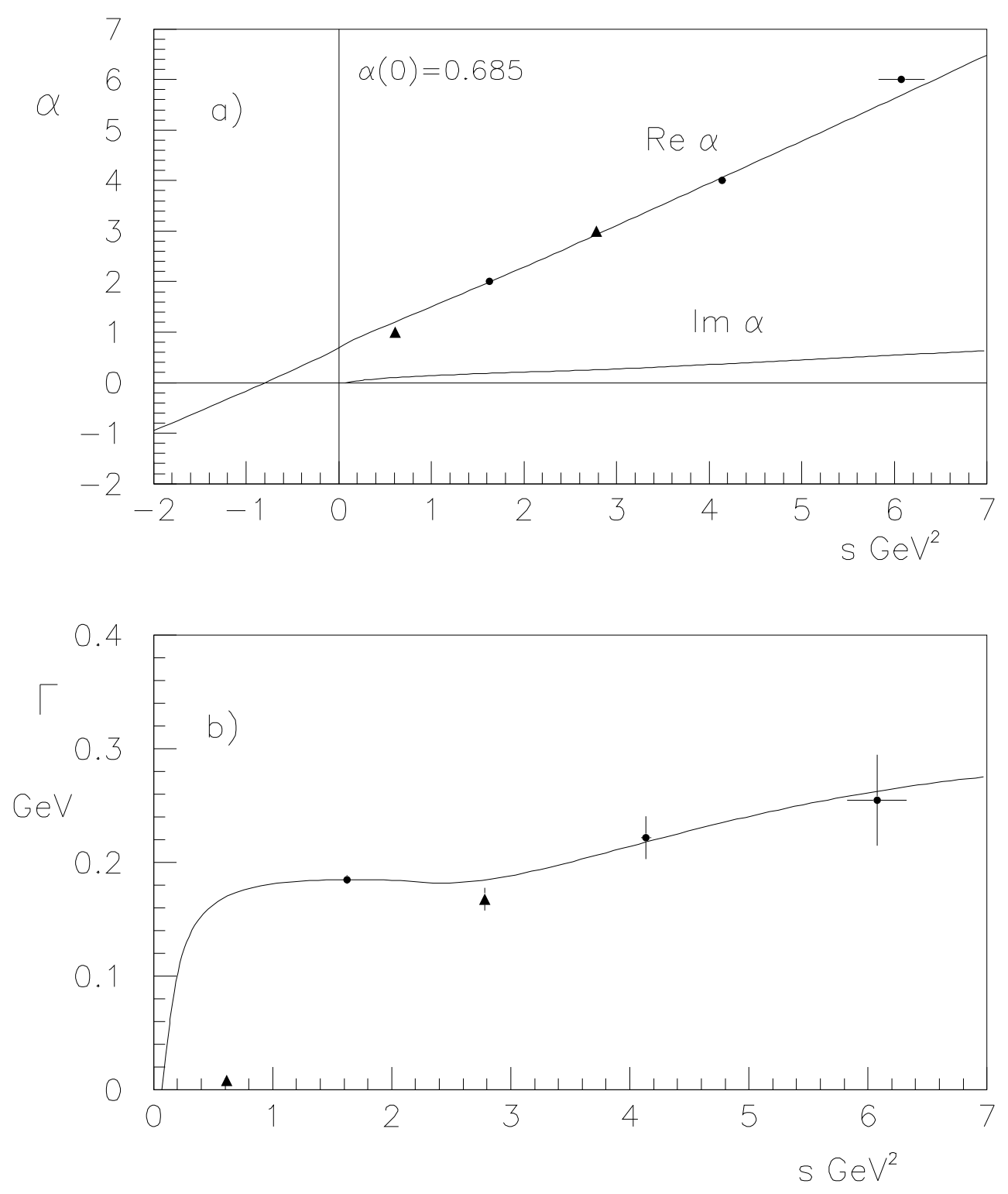

Figure 2: (a) $\mathcal{R} e \alpha(s), \mathcal{I} m \alpha(s)$ for the (non-degenerate) $f$ trajectory; (b) $\Gamma(s)$ for the $f$ trajectory. In both figures triangles symbolize $\omega(782)$ and $\omega_{3}(1670)$, these resosonances have not been included in the fit. 
Let us first consider the $\rho$ and $a_{2}$ trajectories. In fact only the EXD $\rho-a_{2}$ trajectory provides for a large set of input data: ten plus two, which need confirmation. In our model $\rho$ and $a_{2}$ have different thresholds, with different G-parity, and exchange degeneracy is necessarily broken. Experimentally however the breaking is small and not easily detectable in the real part. As a first attempt, we keep all the resonances on an EXD $\rho-a_{2}$ trajectory and fit them with five correction cycles. Data for the resonances $\rho(770), a_{2}(1320), \rho_{3}(1690), a_{4}(2040), \rho_{5}(2350)$ and $a_{6}(2450)$ are taken from the Review of Particle Physics [22] and the zeroth order trajectory is written as $\mathcal{R} e \alpha_{\rho-a_{2}}\left(s_{n}\right) \equiv \lambda_{n} \approx 0.482+0.874 s_{n}$. Notice that $a_{6}(2450)$ needs confirmation and $\rho_{5}(2350)$ has been omitted from the summary table. Thresholds $s_{1}$ and $s_{2}$ are fixed as explained above, $s_{x}$ prefers to stay as large as possible and is fixed at 30 $\mathrm{GeV}^{2}$. In the output the intercept of the degenerate $\rho-a_{2}$ trajectory increased to $\alpha_{\rho-a_{2}}(0) \simeq 0.491$ while $c_{1} \simeq 0.140, c_{2} \simeq 0.902, c_{x} \simeq 28.031$. Figs. $\mathbb{1}(\mathrm{a}, \mathrm{b})$ show, as continuous lines, $\operatorname{Re} \alpha(s), \operatorname{Im} \alpha(s)$ and $\Gamma(s)$ for the exchange degenerate $\rho-a_{2}$. In agreement with Eq. (9), the widths of the resonances are affected to a larger extent by a breaking of EXD. To substantiate this statement we performed a new fit, keeping only the $\rho$ resonances, with the same thresholds. While the real part of the trajectory does not change appreciably, we obtain a good agreement for the widths as shown by the dashed line in Fig. 1(b).

The study of the $f$-trajectory becomes, in this approach, important under many aspects. Analyses of total cross sections depend crucially on its intercept. Moreover, EXD must be badly broken since attempts to describe together $f$ and $\omega$ in this model failed, supporting recent findings [9, 10]. The EXD violation can be understood by noticing that the width of the $\omega(782)$ is only four percent of the width of the $f(1270)$, and it will be difficult to find an analytic function satisfying these 


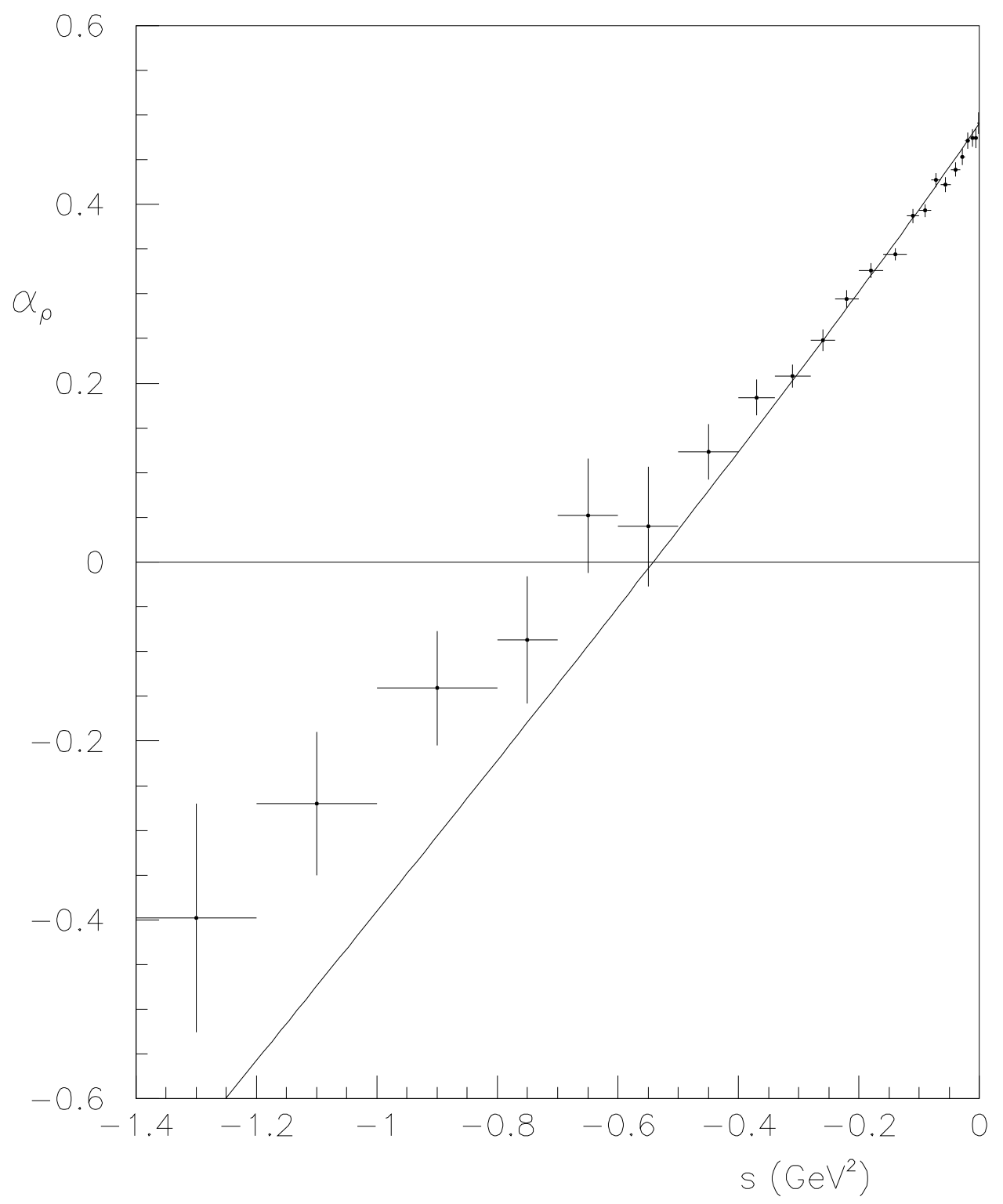

Figure 3: Full line is the degenerate $\rho-a_{2}$ trajectory for negative values of $s$, in the scattering region. Points with error bars show the effective trajectory obtained by fitting pion charge-exchange scattering data [2]]. 
constraints. Starting from the resonances $f_{2}(1270), f_{4}(2050)$ and $f_{6}(2510)$, which needs confirmation [22], and the zeroth order trajectory $\alpha_{f}(s)=0.697+0.801 \cdot s$, with errors quoted in [9], we get, with the higher threshold $s_{x}=\left(2 m_{f_{2}}\right)^{2} \simeq 6.51$ $\mathrm{GeV}^{2}$, a final intercept $\alpha_{f}(0)=0.685$. At the end of 5 th cycle, the parameters are $c_{1} \simeq 0.155, c_{2} \simeq 0.247$ and $c_{x}=8.561$. Figs. $2(\mathrm{a}, \mathrm{b})$ show again the $\mathcal{R} e \alpha(s)$, $\mathcal{I} m \alpha(s)$ and the width, $\Gamma_{f}(s)$, compared with the experimental points. In Fig. 2 triangles symbolize $\omega(782)$ and $\omega_{3}(1670)$ : these resonances are not included in the fit, but their masses and widths are drawn to show once again, that EXD breaking affects more the widths than the real part of the trajectories.

The real part of the degenerate $\rho-a_{2}$ trajectory has been evaluated also for negative values of $s$, in the scattering region. From Eq. (7) a quasi-linear trajectory results with a very small positive curvature. In Fig. 3 we compare our result with an effective trajectory obtained by fitting pion charge-exchange scattering data [20].

Only for $s>-0.4 \mathrm{GeV}^{2}$ the agreement with the experimental points is very good. The curvature of the analytic trajectory has the correct sign, but is not sufficient at large $|s|$. A possible solution at large negative $s$ has been proposed in Ref. [3], where the fit to the existing data [20, 21, 22, 23, 24] of the differential cross section $d \sigma / d t$ for the process $\pi^{-} p \rightarrow \pi^{0} n$ reproduces correctly the experimental data. However, in [3] the trajectory has logarithmic asymptotics and it would be difficult to construct a completely solvable analytic model in this case.

\section{Conclusions}

In this paper we attempt to systematize the light unflavored mesonic trajectories by simultaneous fits to the masses of the resonances and their widths. A deeper 
understanding of EXD plays an important role in this program. In addition to the known properties of the S-matrix, analyticity and unitarity, other assumptions are needed in order to have a completely soluble model. The imaginary part of a trajectory depends on the presence of one or more branch points, related to corresponding thresholds, for positive values of the argument. The first hypothesis is that these thresholds are additive. Moreover, analyticity places an upper bound on the asymptotic behaviour of a trajectory. The second assumption fixes a particular asymptotic form compatible with this constraint. These two assumptions, regarding only the imaginary part of the trajectory, determine the analytic structure of the model.

The parameters of the model, the position of the thresholds and their weight together with the intercept of the trajectory, have been determined for the EXD $\rho-a_{2}$ trajectories and for the $\rho$ and $f$ alone, and the EXD violation has been discussed and explained in the context of the model.

Due to the asymptotic behaviour of the real part, only a finite number of resonances may lie on a given trajectory in this model. However, for the examples considered here the bound on the resonance masses is too high for putting any constraint on a future search of new mesonic states. For the $f$ trajectory in fact, a higher effective threshold will be probably present. In this respect our approach differs from the one in Ref. [19].

The general agreement with the experimental data, in the time-like and spacelike regions, of this simple analytical model makes us hopeful about its capability in predicting properties of mesonic resonances, not yet confirmed or discovered. 


\section{Acknowledgment}

We thanks Enrico Predazzi for stimulating discussions on the subject of this paper.

One of us (L.L.J.) is grateful to the Dipartimento di Fisica dell'Università della Calabria and to the Istituto Nazionale di Fisica Nucleare - Sezione di Padova e Gruppo Collegato di Cosenza for their warm hospitality and financial support. The work of L.L.J. was partly supported by INTAS, grant 97-1696, and CRDF, grant UP1-2119. 


\section{References}

[1] J. Dey et al., Phys. Lett. B365, 157 (1996).

[2] S. Filipponi, G. Pancheri, Y. Srivastava, Phys. Rev. D59, 076003 (1999).

[3] R. Fiore et al, hep-ph/9911503, to be published in the Proceedings of the Bogolyubov Conference (1999).

[4] M.M. Brisudovà, L. Burakovsky and T. Goldman, Phys. Rev. D61, 054013 (2000), hep-ph/0009126.

[5] A.V. Anisovich, V.V. Anisovich and A.V. Sarantsev, Phys. Rev. D62, 051502 $(2000)$.

[6] A. Tang and J.W. Norbury, Phys. Rev. D62, 016006 (2000), hep-ph/0004078.

[7] P. Gauron, B. Nicolescu, Phys. Lett. B486, 71 (2000), hep-ph/0004066.

[8] C. Kontros, J. Kontros, A. Lengyel, hep-ph/0006141.

[9] P. Desgrolard, M. Giffon, E. Martynov, E. Predazzi, hep-ph/0006244.

[10] E. Martynov, hep-ph/9910552, to be published in the Proceedings of the International Conference on Elastic and Difractive Scattering (YIIIth "Blois Workshop") (1999).

[11] B.M. Barbashov, V.N. Pervushin, presented at the New Trend in High-Energy Physics, Proceedings of the Crimean Conference 2000, ed. P.N. Bogolyubov and L.L. Jenkovszky, Kiev, 2000, p. 270.

[12] A.O. Barut and D.E. Zwanziger, Phys. Rev. 127, 974 (1962).

[13] V.N. Gribov and Ya.I. Pomeranchuk, Nucl. Phys. 38, 516 (1962).

[14] R. Taylor, Phys. Rev. 127, 974 (1962). 
[15] R. Oehme, presented at the Strong interactions and high energy physics, Scottish Summer School 1963, ed. R.G. Moorhouse et al., Edinburgh (1964), p. 129.

[16] A. Degasperis and E. Predazzi, Nuovo Cimento A65, 764 (1970).

[17] A.I. Bugrij et al., Fortschritte der Physik, 21, 427 (1973); L.L. Jenkovszky, Riv. Nuovo Cim. 10, 1 (1987).

[18] A.A. Trushevsky, Ukr. Fiz. Zh. 22, 353 (1977).

[19] N.A. Kobylinsky and A.B. Prognimac, Acta Physica Polonica B9, 149 (1978) and earlier references therein.

[20] A.V. Barnes et al., Phys. Rev. Lett. 37, 76 (1976).

[21] A. Erdélyi et al, Table of Integral Transforms (McGraw-Hill, New York, 1953), Vol. II.

[22] D.E. Groom et al., Eur. Phys. J. C15, 1 (2000) and http://pdg.lbl.gov/.

[23] Serpukhov-CERN Collaboration (W.D. Apel et al.), Phys. Lett. B72, 132 (1977); JETP Lett. 26, 502 (1977).

[24] Serpukhov-Brussels-Annecy (LAPP) Collaboration (F. Binon et al.), Z. Phys. C9, 109 (1981). 